\title{
Some Analytical Solitary Wave Solutions for the Generalized q-Deformed Sinh-Gordon Equation: $\partial^{2} \theta / \partial z \partial \xi=\alpha\left[\sinh _{q}\left(\beta \theta^{\gamma}\right)\right]^{p}-\delta$
}

\author{
H. Eleuch $\mathbb{1 D}^{1,2}$ \\ ${ }^{1}$ Institute for Quantum Science and Engineering, Texas A\&M University, College Station, TX 77843, USA \\ ${ }^{2}$ Department of Applied Sciences and Mathematics, College of Arts and Sciences, Abu Dhabi University, Abu Dhabi, UAE
}

Correspondence should be addressed to H. Eleuch; hichemeleuch@yahoo.fr

Received 28 June 2018; Accepted 9 September 2018; Published 25 September 2018

Academic Editor: Boris G. Konopelchenko

Copyright (C) $2018 \mathrm{H}$. Eleuch. This is an open access article distributed under the Creative Commons Attribution License, which permits unrestricted use, distribution, and reproduction in any medium, provided the original work is properly cited.

We introduce the generalized q-deformed Sinh-Gordon equation and derive analytical soliton solutions for some sets of parameters. This new defined equation could be useful for modeling physical systems with violated symmetries.

\section{Introduction}

Dynamical models are the cornerstone of several fields of science and uneven covered in the literature [1-9]. They represent an important research area in mathematics and theoretical physics. Such models are usually based on nonlinear ordinary or partial differential equations. Nonlinearity has generated crucial effects and phenomena not only in the macroscopical systems but also in the microscopical systems governed by quantum physics. We can cite as examples Rogue waves [10-12], bifurcation [13, 14], bistability [1518], chaos [19-22], phase transition [23-26], lasing [27-31], superradiance [32-34], correlations [35-37], squeezing [3841], and solitons [42-48]. A particular interest is focused on solitons due to their potential new application perspectives in several fields of physics and engineering [49-56]. Soliton is a propagating wave solution that conserves its shape and has a particle-like behavior. This propriety could be explained in general by a balance between dispersion and nonlinearity. Several famous nonlinear partial differential equations generate solitons [43, 57-61] such as Korteweg-de Vries equation, nonlinear Schrödinger equation, KadomtsevPetviashvili equation, Sin-Gordon, and Sinh-Gordon equations. The Sinh-Gordon equation has several applications such as in surface theory [62], crystal lattice formation [63], and string dynamics in curved space-time [64]. When the q-deformed hyperbolic function, proposed in the ninetieth of the last century by Arai $[65,66]$, is included in the dynamical system, the symmetry of the system is destroyed and consequently the symmetry of the solution. Recently, several solutions for Schrödinger equation and Dirac equation with q-deformed hyperbolic potential are generated [6772]. q-deformed functions are very promising for modeling atom-trapping potentials or statistical distributions in BoseEinstein condensates $[73,74]$ as well as for exploring vibrational spectra of diatomic molecules $[75,76]$.

In this work, we propose to analyze the propagating wave solutions for a more general form of the Sinh-Gordon equation, the generalized q-deformed Sinh-Gordon equation. As far as we know, this equation is introduced for the first time. We derive for some sets of parameters analytical soliton solutions. The generalized q-deformed Sinh-Gordon equation will open the door for conceiving models of physical systems where the symmetry is absent or violated.

\section{Preliminaries}

Let us first define the generalized q-deformed Sinh-Gordon equation as

$$
\frac{\partial^{2} \theta}{\partial z \partial \xi}=\alpha\left[\sinh _{q}\left(\beta \theta^{\gamma}\right)\right]^{p}-\delta,
$$

where $\alpha, \gamma, p \in \mathbb{R}^{*}, \delta \in \mathbb{R}$, and $\beta \in \mathbb{R}^{+}$. For $\delta / \alpha \geqslant 0$, (1) admits a trivial constant solution

$$
\theta(z, \xi)=\left[\frac{1}{\beta} \ln \left(\left(\frac{\delta}{\alpha}\right)^{1 / p}+\sqrt{\left(\frac{\delta}{\alpha}\right)^{2 / p}+q}\right)\right]^{1 / \gamma} .
$$


It is useful to mention that any first-order polynome $\left(P o_{1}(s)=\right.$ $a_{1} s+a_{0}$; with $\left.a_{1} \neq 0\right)$ in $z$ or in $\xi$ or any combination of two arbitrary first-order polynomes $\left(\alpha_{0} P o_{1}(z)+\alpha_{1} p o_{1}(\xi)\right)$ can not be a solution to the generalized q-deformed Sinh-Gordon equation (1). Equation (1) is also equivalent to

$$
\frac{\partial^{2}\left(\theta_{1}^{\gamma_{1}}\right)}{\partial z \partial \xi}=\alpha\left[\sinh _{q}\left(\beta \theta_{1}\right)\right]^{p}-\delta
$$

where $\theta_{1}=\theta^{\gamma}$ and $\gamma_{1}=1 / \gamma \cdot \sinh _{q}$ is the Arai q-deformed function defined by

$$
\sinh _{q}(x)=\frac{e^{x}-q e^{-x}}{2}
$$

and we have also

$$
\begin{aligned}
& \cosh _{q}(x)=\frac{e^{x}+q e^{-x}}{2}, \\
& \operatorname{sech}_{q}(x)=\frac{1}{\cosh _{q}(x)}, \\
& \tanh _{q}(x)=\frac{e^{x}-q e^{-x}}{e^{x}+q e^{-x}}=\frac{\sinh _{q}(x)}{\cosh _{q}(x)}
\end{aligned}
$$

where $0<q \leq 1$. For $q=1$ we get the standard sinh cosh, sech, and tanh functions. We list below some simple and useful relations for q-deformed functions

$$
\begin{aligned}
& \left(\cosh _{q}(x)\right)^{2}-\left(\sinh _{q}(x)\right)^{2}=q, \\
& \sinh _{q}(-x)=-q \sinh _{1 / q}(x), \\
& \cosh _{q}(-x)=q \cosh _{1 / q}(x), \\
& \frac{d}{d x} \sinh _{q}(x)=\cosh _{q}(x), \\
& \frac{d}{d x} \cosh _{q}(x)=\sinh _{q}(x), \\
& \frac{d}{d x} \tanh { }_{q}(x)=\frac{q}{\left(\cosh _{q}(x)\right)^{2}}=1-\tanh _{q}^{2}(x), \\
& \frac{d}{d x} \operatorname{sech}_{q}(x)=-\tanh _{q}(x) \sec _{q}(x), \\
& \frac{d}{d x} \arcsin _{q}(x)=\frac{1}{\sqrt{q+x^{2}}}, \\
& \frac{d}{d x} \arctan h_{q}(x)=\frac{1}{1-x^{2}},
\end{aligned}
$$

where $\arctan h_{q}$ and $\operatorname{arcsinh} h_{q}$ are, respectively, the inverse functions of $\tanh _{q}$ and $\sinh _{q}$. Since $\tanh _{q}(x)$ and $\sinh _{q}(x)$ vanish at $x=(1 / 2) \ln (q)$ then

$$
\begin{aligned}
\operatorname{arcsinh}_{q}(x) & =\int_{0}^{x} \frac{d x^{\prime}}{\sqrt{q+\left(x^{\prime}\right)^{2}}}+\frac{1}{2} \ln (q) \\
& =\ln \left[x+\sqrt{x^{2}+q}\right], \\
\operatorname{arctanh}_{q}(x) & =\int_{0}^{x} \frac{d x^{\prime}}{1-\left(x^{\prime}\right)^{2}}+\frac{1}{2} \ln (q) \\
& =\frac{1}{2} \ln \left[\frac{q(1+x)}{1-x}\right] ; \quad|x|<1 .
\end{aligned}
$$

It is worth mentioning that the generalized q-deformed SinhGordon equation (1) with $\delta=0$

$$
\frac{\partial^{2} \theta}{\partial z \partial \xi}=\alpha\left[\sinh _{q}\left(\beta \theta^{\gamma}\right)\right]^{p}
$$

can be easily transformed to the generalized q-deformed SinGordon equation defined as

$$
\frac{\partial^{2} \theta}{\partial z \partial \xi}=\alpha\left[\sin _{q}\left(\beta \theta^{\gamma}\right)\right]^{p},
$$

by changing $\theta \longrightarrow(i)^{1 / \gamma} \theta$ and $z \longrightarrow(i)^{(1 / \gamma-p)} z$.

Let us return to (1), by defining the following new normalized coordinates:

$$
\begin{aligned}
& z_{1}=\beta^{1 / 2 \gamma} \sqrt{|\alpha|} z, \\
& \xi_{1}=\beta^{1 / 2 \gamma} \sqrt{|\alpha|} \xi
\end{aligned}
$$

and using

$$
u=\beta^{1 / \gamma} \theta
$$

(1) becomes

$$
\frac{\partial^{2} u}{\partial z_{1} \partial \xi_{1}}= \pm\left(\left[\sinh _{q}\left(u^{\gamma}\right)\right]^{p}-\delta_{1}\right) .
$$

Here \pm is defined by the sign of $\alpha$ and $\delta_{1}=\delta /|\alpha|$. Using the standard transformation

$$
x=a z_{1}+\frac{\xi_{1}}{a}
$$

and

$$
t=a z_{1}-\frac{\xi_{1}}{a}
$$

with $a$ is an arbitrary constant, we obtain then

$$
\frac{\partial^{2} u}{\partial x^{2}}-\frac{\partial^{2} u}{\partial t^{2}}=\left[\sinh _{q}\left(u^{\gamma}\right)\right]^{p}-\delta_{1}
$$


This equation is obtained for positive $\alpha$, and for negative $\alpha$ we obtain the same equation as (27) by interchanging $x \longrightarrow t$ and $t \longrightarrow x$. Equation (27) has two conservative quantities, namely, the total energy $E$ and the momentum $P[77,78]$ defined by

$$
E=\int_{-\infty}^{\infty}\left(\frac{\partial^{2} u}{\partial x^{2}}-\frac{\partial^{2} u}{\partial t^{2}}\right)-F(u) d x
$$

and

$$
P=\frac{1}{2} \int_{-\infty}^{\infty}\left(\frac{\partial u}{\partial t}\right)\left(\frac{\partial u}{\partial x}\right) d x
$$

where

$$
F(u)=\int\left[\sinh _{q}\left(u^{\gamma}\right)\right]^{p} d u-\delta_{1}
$$

\section{Traveling Wave Solution}

In this section we explore the traveling wave solutions of the generalized q-deformed Sinh-Gordon equation (27). We first define a new moving coordinate

$$
\eta=\frac{1}{\sqrt{1-c^{2}}}(x-c t)
$$

so

$$
\begin{aligned}
& \frac{\partial}{\partial t}=-\frac{c}{\sqrt{1-c^{2}}} \frac{\partial}{\partial \eta} \\
& \frac{\partial}{\partial x}=\frac{1}{\sqrt{1-c^{2}}} \frac{\partial}{\partial \eta}
\end{aligned}
$$

$c$ can be interpreted as the speed of the traveling wave in the space-time $(x-t)$. Note that by defining $\eta_{1}=x \pm t$ we get the trivial constant solution for $\delta_{1} \geqslant 0$

$$
u\left(\eta_{1}\right)=\left[\ln \left(\delta_{1}^{1 / p}+\sqrt{\delta_{1}^{2 / p}+q}\right)\right]^{1 / \gamma}
$$

Here we take $c \neq \pm 1$; the traveling wave in the moving frame $\eta$ verifies

$$
\frac{d^{2} u(\eta)}{d \eta^{2}}=\left[\sinh _{q}\left(u^{\gamma}(\eta)\right)\right]^{p}-\delta_{1} .
$$

It is worth mentioning that, for $p=1, \gamma=1, \delta_{1}=0$, and $q=$ $1,(27)$ describes the standard traveling wave of Sinh-Gordon equation. Let us consider now the following particular cases.

3.1. Special Case (1): The Deformed Sinh-Gordon Equation ( $p=$ $1, \gamma=1$, and $\delta_{1}=0$ ). For $=1, \delta_{1}=0$, and $\gamma=1$ (in this case (27) can be identified as a deformed Sinh-Gordon equation). We can multiply both side of (34) by $d u(\eta) / d \eta$ and after the integration we get

$$
\frac{1}{2}\left[\frac{d u(\eta)}{d \eta}\right]^{2}=\cosh _{q}(u(\eta))+c_{1}^{\prime},
$$

so we have an implicit equation for the traveling wave

$$
\int^{u} \frac{d u^{\prime}}{\sqrt{2 \cosh _{q}\left(u^{\prime}\right)+c_{1}}}= \pm\left(\eta+c_{2}^{\prime}\right)
$$

which can be written also as

$$
H(u) \pm \eta=C,
$$

where

$$
H(u)=\int^{u} \frac{d u^{\prime}}{\sqrt{2 \cosh _{q}\left(u^{\prime}\right)+c_{1}}} .
$$

$C$ and $c_{1}$ are free parameters. We can derive general explicit solutions for the considered q-deformed Sinh-Gordon equation. In fact

$$
\begin{aligned}
H(u) & =\int^{u} \frac{d u^{\prime}}{\sqrt{e^{u \prime}+q e^{-u^{\prime}}+c_{1}}} \\
& =\int^{e^{u}} \frac{d y}{\sqrt{y^{3}+q y+c_{1} y^{2}}}
\end{aligned}
$$

and let us first remind the definition of the Weierstrass Elliptic function $\varrho(x)$ :

$$
x=\int_{\varrho\left(x ; g_{2} ; g_{3}\right)}^{\infty} \frac{d w}{\sqrt{4 w^{3}-g_{2} w-g_{3}}} .
$$

so we need to transform the expression of $H(u)$ and in particular the polynomial $P_{3}(y)=y^{3}+c_{1} y^{2}+q y$. By defining $y_{1}=y+c_{1} / 3$ the polynomial $P_{3}(y)$ will be transformed to $(1 / 4)\left(4 y_{1}^{3}+4\left(\left(3 q-c_{1}^{2}\right) / 3\right) y_{1}+4\left(2 c_{1}^{3} / 27-q c_{1} / 3\right)\right)$, where $g_{2}=-4\left(\left(3 q-c_{1}^{2}\right) / 3\right)$ and $g_{3}=-4\left(2 c_{1}^{3} / 27-q c_{1} / 3\right)$, and we have then

$$
H(u)=\int^{e^{u}+c_{1} / 3} \frac{2 d y_{1}}{\sqrt{4 y_{1}^{3}-g_{2} y_{1}-g_{3}}} .
$$

Finally, we obtain the general expression of the soliton in the moving frame $\eta$

$$
u(\eta)=\ln \left[\varrho\left( \pm \frac{1}{2} \eta+c_{2} ; g_{2} ; g_{3}\right)-\frac{c_{1}}{3}\right]
$$

with

$$
\begin{aligned}
& g_{2}=4\left(\frac{c_{1}^{2}-3 q}{3}\right), \\
& g_{3}=4\left(\frac{q c_{1}}{3}-\frac{2 c_{1}^{3}}{27}\right) .
\end{aligned}
$$

$c_{1}$ and $c_{2}$ are free parameters. Let us now study another case. 
3.2. Special Case (2): $\left(p=-2, \gamma=1\right.$, and $\left.\delta_{1}=0\right)$. In this section we shall derive analytical soliton solutions for the case

$$
\frac{d^{2} u(\eta)}{d \eta^{2}}=\frac{1}{\left[\sinh _{q}(u(\eta))\right]^{2}} .
$$

After multiplication of (45) by $d u(\eta) / d \eta$ and integration, we have

$$
\frac{d u(\eta)}{d \eta}= \pm \sqrt{\frac{4}{\left(q-e^{2 u(\eta)}\right)}+c_{1}},
$$

where $c_{1}$ is a free parameter.

Equation (46) can be integrated and we obtain then the following general implicit solutions:

$$
\begin{aligned}
0 & =\sqrt{\frac{q}{4+c_{1} q}} \ln \left[c_{1} q^{2}+4 q\right. \\
& +\sqrt{\left(c_{1} q^{2}+4 q\right)\left(e^{2 u(\eta)}-q\right)\left(c_{1} e^{2 u(\eta)}-c_{1} q-4\right)} \\
& \left.-\left(2+c_{1} q\right) e^{2 u(\eta)}\right]+\frac{1}{\sqrt{c_{1}}} \ln \left[2+c_{1} q-c_{1} e^{2 x}\right. \\
& \left.-\sqrt{c_{1}\left(e^{2 u(\eta)}-q\right)\left(c_{1} e^{2 u(\eta)}-c_{1} q-4\right)}\right] \\
& -\sqrt{\frac{4 q}{4+c_{1} q}} u(\eta) \pm 2 \eta+c_{2} .
\end{aligned}
$$

It is worth mentioning that this equation is valid only for $c_{1} \neq$ $-4 / q$ and $c_{1} \neq 0$.

By choosing $c_{1}=0$ or $c_{1}=-4 / q$ we can generate from direct integration of (46) two other sets of implicit solutions, namely,

$$
\begin{aligned}
& \sqrt{q-e^{2 u(\eta)}}-\sqrt{q} \operatorname{arctanh}\left(\sqrt{\frac{q-e^{2 u(\eta)}}{q}}\right) \pm 2 \eta+c_{2} \\
& \quad=0,
\end{aligned}
$$

and

$$
\begin{aligned}
& \sqrt{q e^{-2 u(\eta)}-1}+\arctan \left[\frac{1}{\sqrt{q e^{-2 u(\eta)}-1}}\right] \pm \frac{2}{\sqrt{q}} \eta+c_{2} \\
& =0 .
\end{aligned}
$$

3.3. Special Case (3): ( $p=2, \gamma=1$, and $\left.\delta_{1}=-q / 2\right)$. Another interesting case to study is for $\left(p=2, \gamma=1\right.$, and $\left.\delta_{1}=-q / 2\right)$, the traveling wave equation is then

$$
\begin{aligned}
\frac{d^{2} u(\eta)}{d \eta^{2}} & =\left[\sinh _{q}(u(\eta))\right]^{2}+\frac{q}{2} \\
& =\frac{1}{2}\left[\cosh _{q^{2}}(2 u(\eta))\right] .
\end{aligned}
$$

Let us first mention that $u=c s t$ is not a solution for (50). By using the transformation

$$
v(\eta)=e^{2 u(\eta)},
$$

we get

$$
2 v(\eta) v^{\prime \prime}(\eta)-2 v^{\prime 2}-v(\eta)^{3}-q^{2} v(\eta)=0 .
$$

In order to solve this nonlinear second-order equation we define

$$
w(v)=\left[\frac{d v(\eta)}{d \eta}\right]^{2}
$$

so

$$
\frac{d^{2} v(\eta)}{d \eta^{2}}=\frac{1}{2} \frac{d w}{d v}(v)
$$

and (52) will be transformed to

$$
v \frac{d w}{d v}(v)-2 w(v)=v^{3}+q^{2} v .
$$

This is a first-order inhomogeneous linear differential equation with the general solution

$$
w(v)=v^{3}+c_{1}^{\prime} v^{2}-q^{2} v .
$$

Here $c_{1}^{\prime}$ is an arbitrary constant. From definition (53) and (56) the expression of the soliton in the implicit form is then

$$
\int^{v(\eta)} \frac{d v}{\sqrt{v^{3}+c_{1} v^{2}-q^{2} v}}= \pm \eta+C,
$$

where $C$ and $c_{1}$ are free parameters. We can write

$$
\begin{aligned}
& \int^{v(\eta)} \frac{d v}{\sqrt{v^{3}+c_{1} v^{2}-q^{2} v}} \\
& =-\int_{v(\eta)+c_{1} / 3} \frac{2 d v_{1}}{\sqrt{4 v_{1}^{3}-g_{4} v_{1}-g_{5}}},
\end{aligned}
$$

where

$$
\begin{aligned}
& g_{4}=4\left(\frac{c_{1}^{2}+3 q^{2}}{3}\right), \\
& g_{5}=-4\left(\frac{q^{2} c_{1}}{3}+\frac{2 c_{1}^{3}}{27}\right),
\end{aligned}
$$

The expression of the solution for (52) is then

$$
v(\eta)=\varrho\left( \pm \frac{1}{2} \eta+c_{2} ; g_{4} ; g_{5}\right)-\frac{c_{1}}{3},
$$

and consequently the general expression of the soliton in the moving frame $\eta=\left(1 / \sqrt{1-c^{2}}\right)(x-c t)$ is

$$
u(\eta)=\ln \left[\sqrt{\varrho\left( \pm \frac{1}{2} \eta+c_{2} ; g_{4} ; g_{5}\right)-\frac{c_{1}}{3}}\right]
$$

where $c_{1}$ and $c_{2}$ are free parameters. 


\section{Conclusion}

We have introduced a generalized q-deformed Sinh-Gordon equation defined by (1) $\left(\partial^{2} \theta / \partial z \partial \xi=\alpha\left[\sinh _{q}\left(\beta \theta^{\gamma}\right)\right]^{p}-\delta\right)$. We have derived general explicit analytical soliton solutions for two sets of parameters, namely, $(p=1, \gamma=1$, and $\delta=0)$ and $(p=2, \gamma=1$, and $\delta=-(q / 2)|\alpha|)$. Furthermore, we have given general implicit soliton solutions for the case: $p=-2$, $\gamma=1$, and $\delta=0$. Future investigations will be focusing on generating more soliton solutions using algebraic methods such as Tanh-method [79], rational expansion method [80], F-expansion method [81], and auxiliary method [82]. The general study of the traveling wave solutions for the generalized q-deformed Sinh-Gordon equation or its asymptotic behavior as well as the analysis of some other proprieties will be for sure very interesting. The usefulness of the generalized q-deformed Sinh-Gordon equation will not be limited to the fields of applied mathematics and mathematical physics but also will open the door for applications in physics where the symmetry of the studied system is absent or violated.

\section{Data Availability}

No data were used to support this study.

\section{Conflicts of Interest}

The author declares that they have no conflicts of interest.

\section{References}

[1] S. H. Strogatz, Nonlinear Dynamics and Chaos: With Applications to Physics, Biology, Chemistry, and Engineering, Westview Press, Boulder, Colorado, 2015.

[2] J. Wang, "Landscape and flux theory of non-equilibrium dynamical systems with application to biology," Advances in Physics, vol. 64, no. 1, pp. 1-137, 2015.

[3] V. Jaksic, D. P. Mandic, K. Ryan, B. Basu, V. Pakrashi, and R. Soc, "A comprehensive study of the delay vector variance method for quantification of nonlinearity in dynamical systems," Royal Society Open Science, vol. 3, Article ID 150493, 2016.

[4] M. Savescu, K. R. Khan, R. W. Kohl, L. Moraru, A. Yildirim, and A. Biswas, "Optical soliton perturbation with improved nonlinear Schrödinger's equation in nano fibers," Journal of Nanoelectronics and Optoelectronics, vol. 8, pp. 208-220, 2013.

[5] R. Sassaman and A. Biswas, "Topological and non-topological solitons of the generalized Klein-Gordon equations," Applied Mathematics and Computation, vol. 215, no. 1, pp. 212-220, 2009.

[6] A. L. Fabian, R. Kohl, and A. Biswas, "Perturbation of topological solitons due to sine-Gordon equation and its type," Communications in Nonlinear Science and Numerical Simulation, vol. 14, no. 4, pp. 1227-1244, 2009.

[7] B. Geranmehr and S. R. Nekoo, "Nonlinear suboptimal control of fully coupled non-affine six-DOF autonomous underwater vehicle using the state-dependent Riccati equation," Ocean Engineering, vol. 96, pp. 248-257, 2015.

[8] X.-Y. Gao, "Looking at a nonlinear inhomogeneous optical fiber through the generalized higher-order variable-coefficient Hirota equation," Applied Mathematics Letters, vol. 73, pp. 143149, 2017.
[9] R. Duan, "Global solutions for a one-dimensional compressible micropolar fluid model with zero heat conductivity," Journal of Mathematical Analysis and Applications, vol. 463, no. 2, pp. 477495, 2018.

[10] Z. Du, B. Tian, H.-P. Chai, Y. Sun, and X.-H. Zhao, "Rogue waves for the coupled variable-coefficient fourth-order nonlinear Schrödinger equations in an inhomogeneous optical fiber," Chaos, Solitons \& Fractals, vol. 10, pp. 90-98, 2018.

[11] L. Liu, B. Tian, Y.-Q. Yuan, and Z. Du, "Dark-bright solitons and semirational rogue waves for the coupled Sasa-Satsuma equations," Physical Review E, Covering Statistical, Nonlinear, Biological, and Soft Matter Physics, vol. 97, Article ID 052217, 2018.

[12] X.-Y. Wu, B. Tian, L. Liu, and Y. Sun, "Rogue waves for a variable-coefficient Kadomtsev-Petviashvili equation in fluid mechanics," Computers \& Mathematics with Applications, vol. 76, no. 2, pp. 215-223, 2018.

[13] Y. A. Kuznetsov, Elements of Applied Bifurcation Theory, vol. 112 of Applied Mathematics Science, Springer, New York, NY, USA, 2nd edition, 1998.

[14] B. Zhilinski, "Quantum bifurcations," in Encyclopedia of Complexity and Systems Science, p. 7135, Springer-Verlag, Berlin, Germany, 2009.

[15] N. N. Rosanov, Spatial Hysteresis and Optical Patterns, Springer Series in Synergetics, Springer-Verlag, Berlin, Germany, 2002.

[16] A. Baas, J. P. Karr, H. Eleuch, and E. Giacobino, "Optical bistability in semiconductor microcavities," Physical Review A: Atomic, Molecular and Optical Physics, vol. 69, no. 2, Article ID 023809, 2004.

[17] E. A. Sete, H. Eleuch, and S. Das, "Controllable nonlinear effects in an optomechanical resonator containing a quantum well," Physical Review A: Atomic, Molecular and Optical Physics, vol. 85, no. 4, Article ID 043824, 2012.

[18] E. A. Sete and H. Eleuch, "Anomalous optical bistability and robust entanglement of mechanical oscillators using twophoton coherence," Journal of the Optical Society of America B, vol. 32, no. 5, pp. 971-982, 2015.

[19] J.-P. Eckmann and D. Ruelle, "Ergodic theory of chaos and strange attractors," Reviews of Modern Physics, vol. 57, no. 3, pp. 617-656, 1985.

[20] M. Sieber and F. Steiner, "Quantum chaos in the hyperbola billiard," Physics Letters A, vol. 148, no. 8-9, pp. 415-420, 1990.

[21] H. Eleuch and A. Prasad, "Chaos and regularity in semiconductor microcavities," Physics Letters A, vol. 376, no. 26-27, pp. 19701977, 2012.

[22] H. Eleuch, A. Prasad, and I. Rotter, "Enhancement of photon intensity in forced coupled quantum wells inside a semiconductor microcavity," Physical Review E: Statistical, Nonlinear, and Soft Matter Physics, vol. 87, no. 2, Article ID 022916, 2013.

[23] V. G. Ivancevic and T. T. Ivancevic, Complex Nonlinearity: Chaos, Phase Transitions, Topology Change and Path Integrals, Springer, New York, NY, USA, 2008.

[24] H. K. Janssen, "On the nonequilibrium phase transition in reaction-diffusion systems with an absorbing stationary state," Zeitschrift für Physik B Condensed Matter, vol. 42, no. 2, pp. 151154, 1981.

[25] D. G. Joshi and M. Vojta, "Nonlinear bond-operator theory and $1 / d$ expansion for coupled-dimer magnets. II. Antiferromagnetic phase and quantum phase transition," Physical Review B: Condensed Matter and Materials Physics, vol. 91, no. 9, Article ID 094405, 2015. 
[26] S. Shrivastava, K. H. Kang, and M. F. Schneider, "Solitary shock waves and adiabatic phase transition in lipid interfaces and nerves," Physical Review E: Statistical, Nonlinear, and Soft Matter Physics, vol. 91, no. 1, Article ID 012715, 2015.

[27] H. Haken, Laser Theory, Springer-Verlag, New York, NY, USA, 1983.

[28] R. Buschlinger, M. Lorke, and U. Peschel, "Light-matter interaction and lasing in semiconductor nanowires: A combined finitedifference time-domain and semiconductor Bloch equation approach," Physical Review B: Condensed Matter and Materials Physics, vol. 91, no. 4, Article ID 045203, 2015.

[29] R. Buschlinger, M. Lorke, and U. Peschel, "Erratum: Lightmatter interaction and lasing in semiconductor nanowires: A combined finite-difference time-domain and semiconductor Bloch equation approach [Phys. Rev. B 91, 045203 (2015)]," Physical Review B: Condensed Matter and Materials Physics, vol. 91, no. 15, Article ID 159903, 2015.

[30] M. Eghbalpour, R. Karimi, S. Batebi, and H. R. Soleimani, "Effect of electron-spin relaxation on optical bistability and lasing without population inversion in a three-level $\mathrm{v}$ type quantum system," Optik - International Journal for Light and Electron Optics, vol. 127, no. 5, pp. 2525-2530, 2016.

[31] E. A. Sete, A. A. Svidzinsky, Y. V. Rostovtsev et al., "Using quantum coherence to generate gain in the XUV and X-ray: gain-swept superradiance and lasing without inversion," IEEE Journal of Selected Topics in Quantum Electronics, vol. 18, no. 1, pp. 541-553, 2012.

[32] M. Gross and S. Haroche, "Superradiance: An essay on the theory of collective spontaneous emission," Physics Reports, vol. 93, no. 5, pp. 301-396, 1982.

[33] E. A. Sete, A. A. Svidzinsky, H. Eleuch, Z. Yang, R. D. Nevels, and M. O. Scully, "Correlated spontaneous emission on the Danube," Journal of Modern Optics, vol. 57, no. 14-15, pp. 13111330, 2010.

[34] C. Svetina, N. Mahne, L. Raimondi et al., "Photon transport of the superradiant TeraFERMI THz beamline at the FERMI free-electron laser," Journal of Synchrotron Radiation, vol. 23, pp. 106-110, 2016.

[35] P. L. Lai and C. Fyfe, "Kernel and nonlinear canonical correlation analysis," International Journal of Neural Systems, vol. 10, 377, no. 5, p. 365, 2000.

[36] K. Berrada, M. El Baz, H. Eleuch, and Y. Hassouni, "A comparative study of negativity and concurrence based on spin coherent states," International Journal of Modern Physics C, vol. 21, no. 3, pp. 291-305, 2010.

[37] H. Eleuch, S. Guérin, and H. R. Jauslin, "Effects of an environment on a cavity-quantum-electrodynamics system controlled by bichromatic adiabatic passage," Physical Review A: Atomic, Molecular and Optical Physics, vol. 85, no. 1, Article ID 013830, 2012.

[38] K. Kasai, G. Jiangrui, and C. Fabre, "Observation of squeezing using cascaded nonlinearity," EPL (Europhysics Letters), vol. 40, no. 1 , article 25, 1997.

[39] P. D. Drummond, Quantum Squeezing, Springer Series on Atomic, Optical, and Plasma Physics, Springer, New York, NY, USA, 2010.

[40] Y. A. Korkmaz and C. Bulutay, "Nuclear spin squeezing via electric quadrupole interaction," Physical Review A: Atomic, Molecular and Optical Physics, vol. 93, no. 1, Article ID 013812, 2016.
[41] E. Giacobino, J.-P. Karr, G. Messin, H. Eleuch, and A. Baas, "Quantum optical effects in semiconductor microcavities," Comptes Rendus Physique, vol. 3, no. 1, pp. 41-52, 2002.

[42] X.-H. Zhao, B. Tian, J. Chai, X.-Y. Wu, and Y.-J. Guo, "Multisoliton interaction of a generalized Schrödinger-Boussinesq system in a magnetized plasma," The European Physical Journal Plus, vol. 132, article 192, 2017.

[43] E. Infeld and G. Rowlands, Nonlinear Waves, Solitons and Chaos, Cambridge University Press, Cambridge, UK, 2000.

[44] H. Eleuch and R. Bennaceur, "An optical soliton pair among absorbing three-level atoms," Journal of Optics A: Pure and Applied Optics, vol. 5, no. 5, pp. 528-533, 2003.

[45] D. S. Kharenko, A. E. Bednyakova, E. V. Podivilov, M. P. Fedoruk, A. Apolonski, and S. A. Babin, "Cascaded generation of coherent Raman dissipative solitons," Optics Expresss, vol. 41, no. 1, pp. 175-178, 2016.

[46] A. Biswas, D. Milovic, and A. Ranasinghe, "Solitary waves of Boussinesq equation in a power law media," Communications in Nonlinear Science and Numerical Simulation, vol. 14, no. 11, pp. 3738-3742, 2009.

[47] G. Ebadi, A. H. Kara, M. D. Petkovic, A. Yildirim, and A. Biswas, "Solitons and conserved quantities of the Ito equation," Proceedings of the Romanian Academy - Series A: Mathematics, Physics, Technical Sciences, Information Science, vol. 13, no. 3, pp. 215-224, 2012.

[48] R. Sassaman, A. Heidari, and A. Biswas, "Topological and nontopological solitons of nonlinear Klein-Gordon equations by He's semi-inverse variational principle," Journal of The Franklin Institute, vol. 347, no. 7, pp. 1148-1157, 2010.

[49] Y. S. Kivshara and B. Luther-Davies, "Dark optical solitons: physics and applications," Physics Reports, vol. 298, no. 2-3, pp. 81-197, 1998.

[50] M. J. Ablowitz, G. Biondini, and L. A. Ostrovsky, "Optical solitons: perspectives and applications," Chaos: An Interdisciplinary Journal of Nonlinear Science, vol. 10, no. 3, pp. 471-474, 2000.

[51] N. Boutabba, H. Eleuch, and H. Bouchriha, "Thermal bath effect on soliton propagation in three-level atomic system," Synthetic Metals, vol. 159, no. 13, pp. 1239-1243, 2009.

[52] R. Hao, "Optical soliton control in inhomogeneous nonlinear media with the parity-time symmetric potentials," Optics Communications, vol. 338, pp. 265-268, 2015.

[53] A. Biswas, "Dispersion-managed solitons in optical fibres," Journal of Optics A: Pure and Applied Optics, vol. 4, no. 1, article $84,2002$.

[54] M. Savescu, A. H. Bhrawy, A. A. Alshaery et al., "Optical solitons in nonlinear directional couplers with spatio-temporal dispersion," Journal of Modern Optics, vol. 61, no. 5, pp. 441-458, 2014.

[55] J. V. Guzman, E. M. Hilal, A. A. Alshaery et al., "Thirring optical solitons with spatio-temporal dispersion," Proceedings of The Romanian Academy, Series A, vol. 16, no. 1, pp. 41-46, 2015.

[56] H. Triki, A. H. Kara, A. Bhrawy, and A. Biswas, "Soliton Solution and Conservation Law of Gear-Grimshaw Model for Shallow Water Waves," Acta Physica Polonica A, vol. 125, no. 5, pp. 10991106, 2014.

[57] A. H. Bhrawy, M. A. Abdelkawy, and A. Biswas, "Cnoidal and snoidal wave solutions to coupled nonlinear wave equations by the extended Jacobi's elliptic function method," Communications in Nonlinear Science and Numerical Simulation, vol. 18, no. 4, pp. 915-925, 2013. 
[58] A. H. Bhrawy, M. A. Abdelkawy, S. Kumar, and A. Biswas, "Solitons and other solutions to Kadomtsev-Petviashvili equation of B-type," Romanian Journal of Physics, vol. 58, no. 7-8, pp. 729748, 2013.

[59] G. Ebadi, N. Y. Fard, A. H. Bhrawy et al., "Solitons and other solutions to the (3+1)-dimensional extended KadomtsevPetviashviliequation with power law nonlinearity," Romanian Reports in Physics, vol. 65, no. 1, pp. 27-62, 2013.

[60] A. Biswas, A. H. Bhrawy, M. A. Abdelkawy, A. A. Alshaery, and E. M. Hilal, "Symbolic computation of some nonlinear fractional differential equations," Romanian Reports in Physics, vol. 59, no. 5-6, pp. 433-442, 2014.

[61] A. Sardar, K. Ali, S. Tahir et al., "Dispersive optical solitons in nanofibers with Schrödinger-Hirota equation," Journal of Nanoelectronics and Optoelectronics, vol. 11, no. 3, pp. 382-387, 2016.

[62] L. Hauswirth, R. Sa Earp, and E. Toubiana, "Associate and conjugate minimal immersions in $M \times R$," The Tohoku Mathematical Journal, vol. 60, no. 2, pp. 267-286, 2008.

[63] M. J. Ablowitz and P. A. Clarkson, Solitons, Nonlinear Evolution Equations and Inverse Scattering, Cambridge University Press, Cambridge, UK, 1991.

[64] A. L. Larsen and N. Sánchez, "sinh-GORdon, cosh-GORdon, and Liouville equations for strings and multistrings in constant curvature spacetimes," Physical Review D: Particles, Fields, Gravitation and Cosmology, vol. 54, no. 4, pp. 2801-2807, 1996.

[65] A. Arai, "Exactly solvable supersymmetric quantum mechanics," Journal of Mathematical Analysis and Applications, vol. 158, no. 1, pp. 63-79, 1991.

[66] A. Arai, "Exact solutions of multi-component nonlinear Schrödinger and Klein-Gordon equations in two-dimensional space-time," Journal of Physics A: Mathematical and General, vol. 34, no. 20, pp. 4281-4288, 2001.

[67] B. J. Falaye, K. J. Oyewumi, and M. Abbas, "Exact solution of Schrödinger equation with $q$-deformed quantum potentials using Nikiforov-Uvarov method," Chinese Physics B, vol. 22, no. 11, Article ID 110301, 2013.

[68] H. Yılmaz, D. Demirhan, and F. Büyükkılıç, "Solution of the Schrödinger equation for two q-deformed potentials by the SWKB method," Journal of Mathematical Chemistry, vol. 47, article 539, 2010.

[69] M. Sebawe Abdalla, H. Eleuch, and T. Barakat, "Exact analytical solutions of the wave function for some q-deformed potentials," Reports on Mathematical Physics, vol. 71, no. 2, pp. 217-229, 2013.

[70] M. Sebawe Abdalla and H. Eleuch, "Exact analytic solutions of the Schrödinger equations for some modified q-deformed potentials," Journal of Applied Physics, vol. 115, no. 23, Article ID 234906, 2014.

[71] M. Eshghi and H. Mehraban, "Solution of the Dirac equation with position-dependent mass for q-parameter modified pöschl-teller and coulomb-like tensor potential," Few-Body Systems, vol. 52, no. 1-2, pp. 41-47, 2012.

[72] A. Kurniawan, A. Suparmi, and C. Cari, "Approximate analytical solution of the Dirac equation with $q$-deformed hyperbolic Pöschl-Teller potential and trigonometric Scarf II non-central potential," Chinese Physics B, vol. 24, no. 3, Article ID 030302, 2015.

[73] Q. J. Zeng, Z. Cheng, and J. H. Yuan, "Bose-Einstein condensation of a $q$-deformed boson system in a harmonic potential trap," Physica A: Statistical Mechanics and its Applications, vol. 3, pp. 563-571, 391.
[74] Y. Shu, J. Chen, and L. Chen, "Bose-Einstein condensation of a q-deformed ideal Bose gas," Physics Letters A, vol. 292, no. 6, pp. 309-314, 2002.

[75] S. M. Ikhdair, "Rotation and vibration of diatomic molecule in the spatially-dependent mass Schrödinger equation with generalized q-deformed Morse potential," Chemical Physics, vol. 361, no. 1-2, pp. 9-17, 2009.

[76] D. Bonatsos, E. N. Argyres, and P. P. Raychev, " $\mathrm{SU}_{q}(1,1)$ description of vibrational molecular spectra," Journal of Physics A: Mathematical and General, vol. 24, no. 8, pp. L403-L408, 1991.

[77] M. Dehghan and A. Ghesmati, "Application of the dual reciprocity boundary integral equation technique to solve the nonlinear Klein-Gordon equation," Computer Physics Communications, vol. 181, no. 8, pp. 1410-1418, 2010.

[78] S. Jiménez and L. Vázquez, "Analysis of four numerical schemes for a nonlinear Klein-Gordon equation," Applied Mathematics and Computation, vol. 35, no. 1, pp. 61-94, 1990.

[79] A.-M. Wazwaz, "The tanh method: exact solutions of the sineGORdon and the sinh-GORdon equations," Applied Mathematics and Computation, vol. 167, no. 2, pp. 1196-1210, 2005.

[80] Q. Wang, Y. Chen, and H. Zhang, "A new Riccati equation rational expansion method and its application to $(2+1)$-dimensional Burgers equation," Chaos, Solitons \& Fractals, vol. 25, no. 5, pp. 1019-1028, 2005.

[81] J. L. Zhang, M. L. Wang, Y. M. Wang, and Z. D. Fang, "The improved F-expansion method and its applications," Physics Letters A, vol. 350, no. 1-2, pp. 103-109, 2006.

[82] A. Sirendaoreji, "Auxiliary equation method and new solutions of Klein-Gordon equations," Chaos, Solitons \& Fractals, vol. 31, no. 4, pp. 943-950, 2007. 


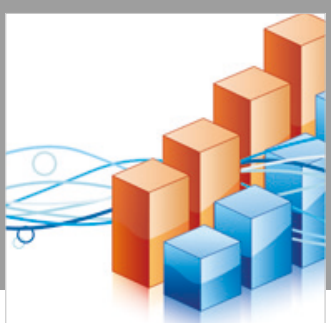

Advances in

Operations Research

\section{-n-m}
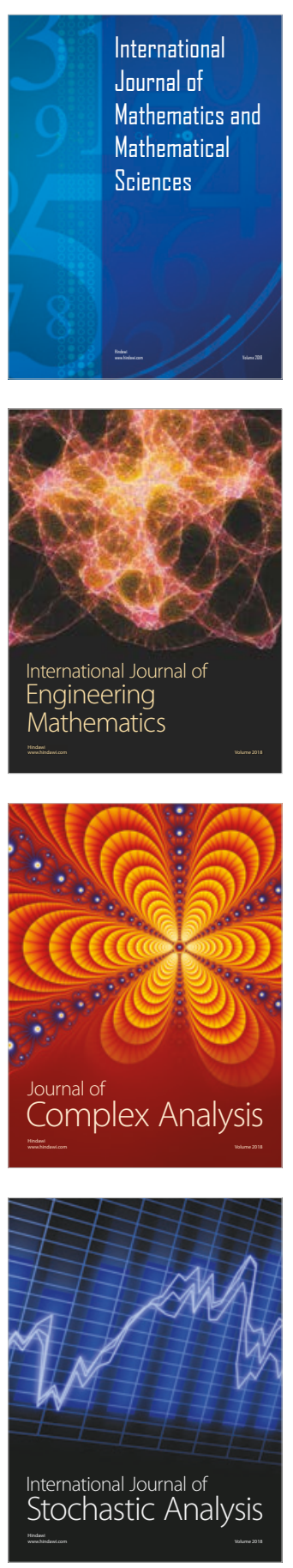
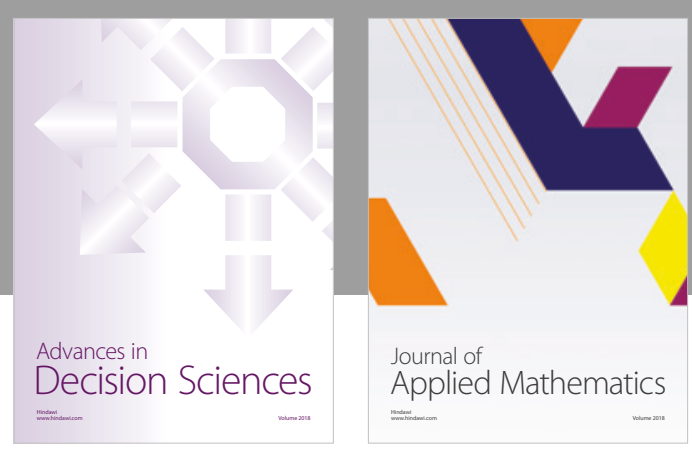

Journal of

Applied Mathematics
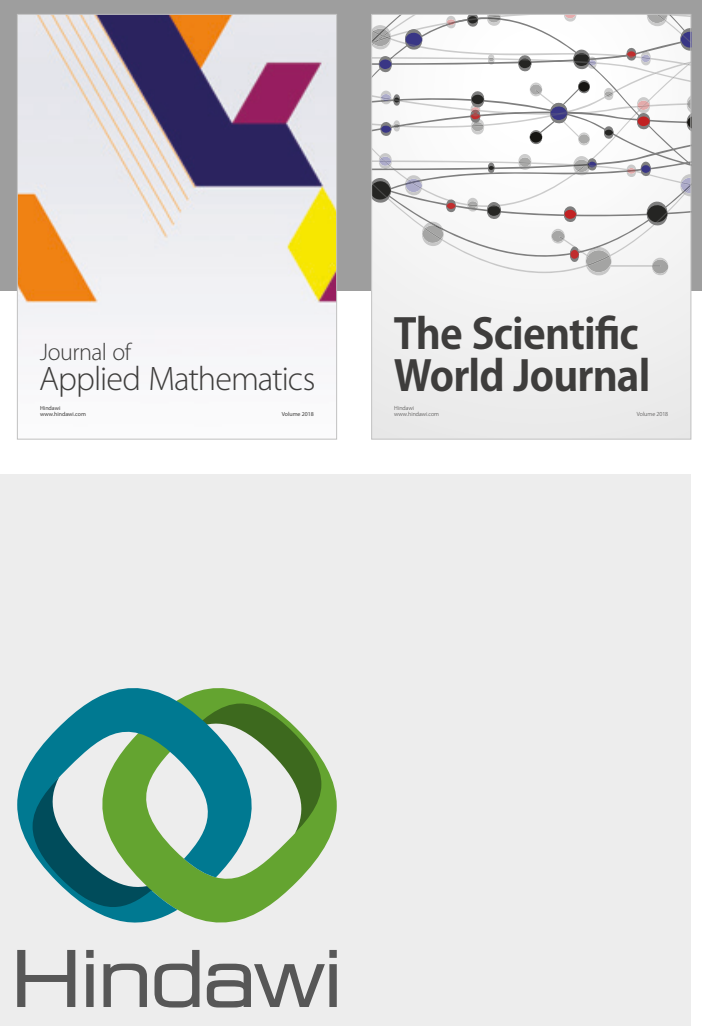

Submit your manuscripts at

www.hindawi.com

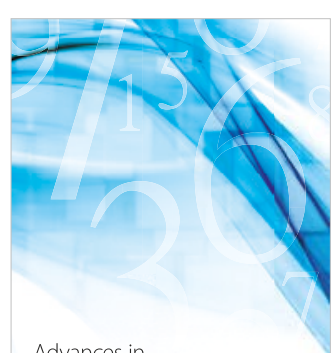

Advances in
Numerical Analysis
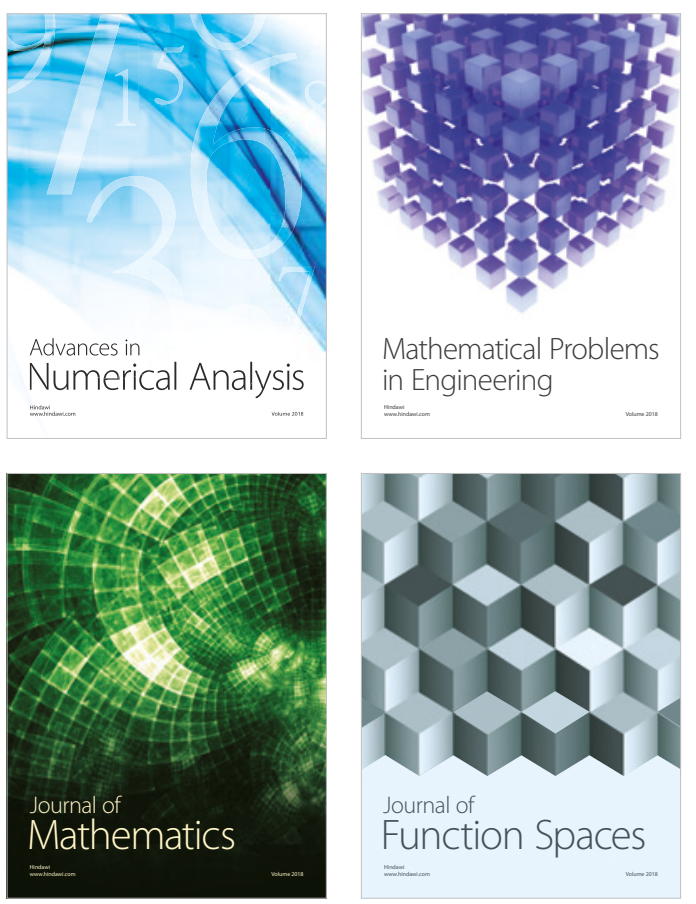

Mathematical Problems in Engineering

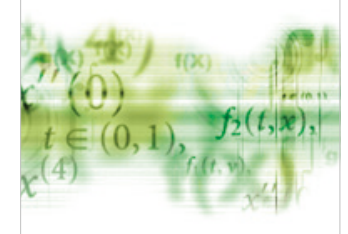

International Journal of

Differential Equations

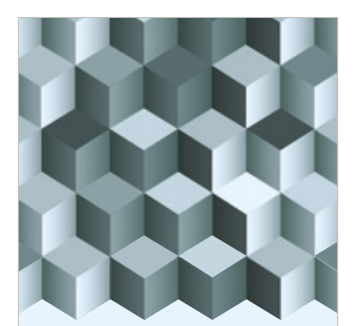

Journal of

Function Spaces

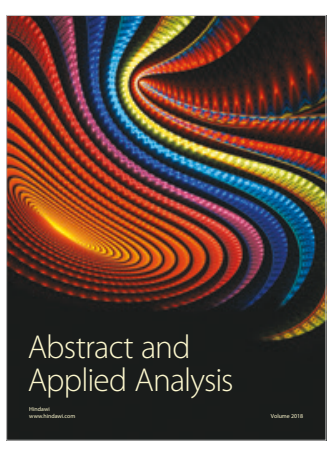

The Scientific

World Journal

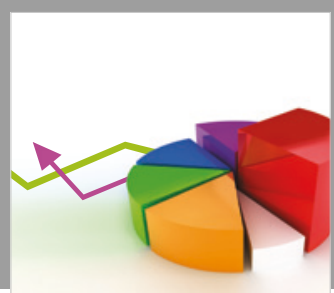

Journal of

Probability and Statistics
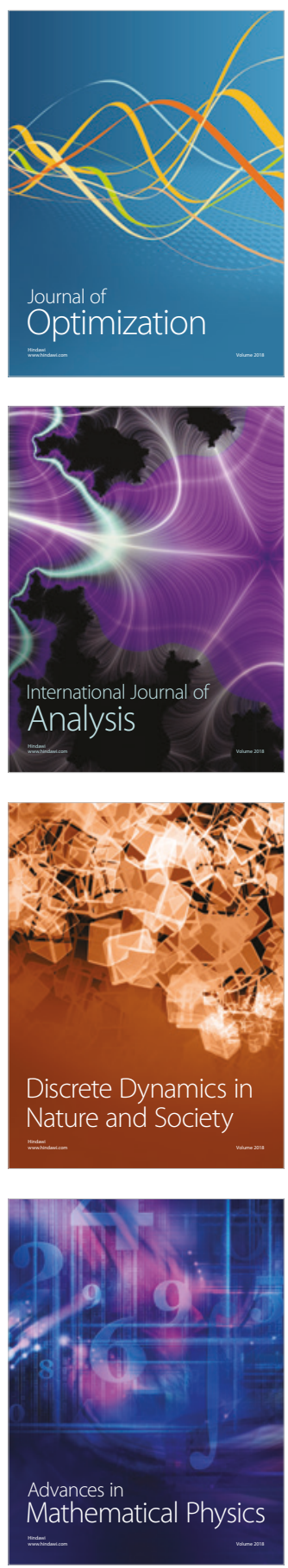